\title{
Hydrolytic activities of artificial nanocellulose synthesized via phosphorylase-catalyzed enzymatic reactions
}

\author{
Takeshi Serizawa $^{1}$, Mari Kato ${ }^{1}$, Hiromichi Okura ${ }^{1}$, Toshiki Sawada ${ }^{1}$ and Masahisa Wada ${ }^{2}$ \\ Nature-based nanocelluloses with the cellulose I allomorph are attractive one-dimensional nanomaterials because of their mass \\ productivity and unique physicochemical properties. Our previous studies demonstrated that the activated ester, monophosphate \\ and amide linkages of small organic substrates were hydrolyzed on the surface of nanocrystalline cellulose (NCC), which is a \\ nature-based nanocellulose. However, fundamental knowledge of the hydrolytic activities of nanocelluloses is limited. In this \\ study, artificial sheet-like nanocelluloses composed of cellulose oligomers with the cellulose II allomorph were synthesized by \\ phosphorylase-catalyzed enzymatic reactions, and their hydrolytic activities against ester substrates were characterized. The \\ as-prepared nanocelluloses exhibited relatively low hydrolytic activities. However, distorted and smaller nanocelluloses with larger \\ surface areas, which were prepared by sonication-based mechanical treatment of the as-prepared nanocelluloses, exhibited \\ significantly greater hydrolytic activities. These enzymatically synthesized artificial nanocelluloses exhibited hydrolytic activities, \\ even though the molecular weights of the component cellulose, allomorphs, and morphologies were different from those of \\ nature-based NCC.
}

Polymer Journal (2016) 48, 539-544; doi:10.1038/pj.2015.125; published online 13 January 2016

\section{INTRODUCTION}

Nature-based nanocelluloses have gained considerable attention as relatively new one-dimensional crystalline cellulose nanomaterials with nanometer-sized widths and high specific surface areas because of their mass productivity and unique physicochemical properties. ${ }^{1-5}$ Nanocelluloses are classified into subcategories, such as microfibrillated cellulose, nanocrystalline cellulose (NCC) and bacterial nanocellulose, according to their production processes. ${ }^{6}$ Combinations of chemical, enzymatic, and mechanical treatments of raw materials, ${ }^{7}$ acid hydrolysis of raw materials ${ }^{1,8}$ and cultivation of bacteria ${ }^{9}$ can be employed to produce the three types of nanocelluloses, respectively. Nanocelluloses are chemically stable, mechanically stiff and allow for surface chemical modification. Therefore, their potential applications as reinforcing agents of composite materials have been investigated. ${ }^{6}$ On the other hand, our previous studies have revealed that ester, monophosphate and even amide linkages of small organic or biorelated molecules are hydrolyzed on the surface of HCl-treated NCC under aqueous mild conditions. ${ }^{10,11}$ This enzyme-like property of NCC can extend its applicability to many technological fields. However, the molecular-level mechanism of the hydrolytic reactions remains unclear. Therefore, gaining fundamental insight into the hydrolytic activities of other cellulose-based nanomaterials is necessary.
To artificially design and control the chemical, crystalline and morphological structures of cellulose, a one-step and environmentally friendly synthesis of cellulose based on enzymatic reactions under aqueous mild conditions has been investigated. ${ }^{12-15}$ For example, when $\alpha$-D-glucose 1 -phosphate $(\alpha \mathrm{G} 1 \mathrm{P})$ monomers were propagated to D-glucose primers by cellodextrin phosphorylase (CDP) through glycosylation reactions, the synthesized cellulose oligomers (that is, cellodextrins) with an average degree of polymerization (DP) of nine formed crystalline two-dimensional nanosheets with a thickness of $4.5 \mathrm{~nm} .{ }^{16}$ The cellulose oligomers in the artificial nanocelluloses with the anti-parallel cellulose II allomorph were aligned perpendicular to the sheet surface, exposing the reducing and non-reducing terminals to the surface. Significantly, artificial nanocelluloses have different chemical, crystalline and morphological structures from nature-based nanocelluloses, which are obtained from natural sources. In fact, nature-based nanocelluloses have much greater DP, cellulose I allomorph with parallel alignment, and fibrous morphologies with fewer reducing and non-reducing terminals on the cellulose chains. ${ }^{1-5}$ Therefore, the hydrolytic activities of these artificial nanocelluloses against organic substrates need to be investigated.

In this study, the hydrolytic activities of artificial nanocelluloses synthesized via CDP-catalyzed enzymatic reactions were quantitatively

\footnotetext{
${ }^{1}$ Department of Organic and Polymeric Materials, Graduate School of Science and Engineering, Tokyo Institute of Technology, Tokyo, Japan and ${ }^{2}$ Division of Forest and Biomaterials Science, Graduate School of Agriculture, Kyoto University, Kyoto, Japan and Department of Plant and Environmental New Resources, College of Life Sciences, Kyung Hee University, Seoul, Republic of Korea

Correspondence: Professor T Serizawa, Department of Organic and Polymeric Materials, Graduate School of Science and Engineering, Tokyo Institute of Technology, 2-12-1-H121 Ookayama, Meguro-ku, Tokyo 152-8550, Japan.

E-mail: serizawa@polymer.titech.ac.jp

Received 27 October 2015; revised 19 November 2015; accepted 19 November 2015; published online 13 January 2016
} 
investigated using a model ester substrate (that is, p-nitrophenyl acetate, PNP) (Figure 1). In addition, the effect of the sonication-based mechanical treatment of the as-prepared nanocelluloses on its hydrolytic activities was characterized in detail. Relatively low but credible hydrolytic activities of the as-prepared nanocelluloses were observed, and then, substantially increased hydrolytic activities were observed for the mechanically treated nanocelluloses with smaller sizes and smaller DP.

\section{EXPERIMENTAL PROCEDURE}

\section{Materials}

$\alpha \mathrm{G} 1 \mathrm{P}$ disodium salt $\mathrm{n}$-hydrate, cellohexaose, and $p$-nitroacetanilide were purchased from Wako Pure Chemical Industries (Osaka, Japan). EM stainer was purchased from Nissin EM Corporation (Tokyo, Japan). All of the other reagents were purchased from Nacalai Tesque (Kyoto, Japan). Ultrapure water with more than $18.2 \mathrm{M} \Omega \mathrm{cm}$ was supplied by a Milli-Q Advantage A-10 (Merck Millipore, Darmstadt, Germany) and used for all of the experiments.

\section{Enzymatic reactions for nanocellulose synthesis}

CDP preparation and CDP-catalyzed enzymatic reactions for nanocellulose synthesis were performed according to our previously reported protocols. ${ }^{17}$ Briefly, CDP from Clostridium thermocellum YM4 was prepared using an Escherichia coli BL21-Gold(DE3) strain containing a plasmid including the $c d p$ gene and purified using a Ni-NTA column (GE Healthcare, Tokyo, Japan) with fused His-tag. The $\alpha \mathrm{G} 1 \mathrm{P}$ monomers $(200 \mathrm{~mm})$ and D-glucose primers $(50 \mathrm{~mm})$ were incubated with $\mathrm{CDP}\left(0.2 \mathrm{U} \mathrm{ml}^{-1}\right)$ in $500 \mathrm{~mm}$ 4-(2-hydroxyethyl)-1piperazineethanesulfonic acid (HEPES) buffer ( $\mathrm{pH} 7.5$ ) at $60^{\circ} \mathrm{C}$ for 3 days. After incubation, the water-insoluble products were washed with ultrapure water via centrifugation at more than 3500 r.p.m. and redispersion cycles to eliminate $99.999 \%$ of the reaction solution, and then, these products were heated at $100^{\circ} \mathrm{C}$ for $10 \mathrm{~min}$ to inactivate the residual $\mathrm{CDP}$. The resulting dispersions were stored at $4{ }^{\circ} \mathrm{C}$ before use. The nanocellulose concentrations were estimated by evaporating the water at $105^{\circ} \mathrm{C}$ for $48 \mathrm{~h}$

\section{Structural analysis of nanocelluloses}

For transmission electron microscopy (TEM) observations, a collodion-coated copper EM grid (Nissin EM Corporation) was placed on aqueous dispersions of the products for $1 \mathrm{~h}$ and then placed on a droplet of ultrapure water. The sample was negatively stained with EM stainer. The prepared grid was dried in a desiccator for at least $12 \mathrm{~h}$. The TEM images were recorded on a Hitachi H-7650 Zero A microscope (Hitachi High-Technologies, Tokyo, Japan) operated at $100 \mathrm{kV}$. The ${ }^{1} \mathrm{H}$ nuclear magnetic resonance (NMR) spectra of the lyophilized products $\left(3(\mathrm{w} / \mathrm{v}) \%\right.$ in $4 \% \mathrm{NaOD} / \mathrm{D}_{2} \mathrm{O}$ were obtained on a Bruker DPX-300 spectrometer (Bruker Biospin, Yokohama, Japan) operated at $300 \mathrm{MHz}$. The chemical shifts were recorded relative to $\mathrm{H}_{2} \mathrm{O}(\delta$ 4.79). The matrix-assisted laser desorption/ionization time-of-flight mass spectra were obtained on a Biotech Aximap Performance (Shimadzu, Kyoto, Japan) using a matrix consisting of 2,5-dihydroxybenzoic acid. The attenuated total reflectionFourier transform infrared absorption spectra of the lyophilized products were obtained on a JASCO FT/IR-4100 spectrometer (JASCO Corporation, Tokyo, Japan) with a cumulative number of 100 under ambient conditions and a

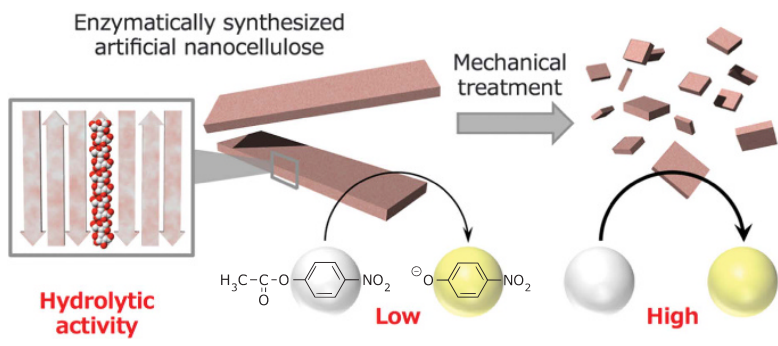

Figure 1 Schematic illustration of this study. resolution of $2.0 \mathrm{~cm}^{-1}$. The wide-angle X-ray diffraction measurements of the lyophilized products were performed under ambient conditions using a Mini Flex 600 (Rigaku, Tokyo, Japan) with $\mathrm{Cu}-\mathrm{K} \alpha$ radiation $(\lambda=0.1542 \mathrm{~nm})$.

\section{Analysis of hydrolytic activities}

Unless otherwise stated, $3 \mu \mathrm{l}$ of the substrate solution $(10 \mathrm{~mm})$ in dimethylsulfoxide with PNP (a standard substrate) and p-nitroacetanilide or in ultrapure water with disodium $p$-nitrophenyl phosphate was added to $297 \mu \mathrm{l}$ of $10 \mathrm{~mm}$ HEPES buffer ( $\mathrm{pH} 7.4$ ) containing $0.5(\mathrm{w} / \mathrm{v}) \%$ of the nanocellulose (the final concentration of substrates was $100 \mu \mathrm{M}$ ) in a $1.5 \mathrm{ml}$ polypropylene tube. Then, a rotator (15 r.p.m.) was employed to mechanically stir the reaction solutions with twice tapping per round at $30^{\circ} \mathrm{C}$ for $7.5 \mathrm{~h}$. After the reaction, the nanocelluloses were precipitated by centrifugation at 15000 r.p.m. for $5 \mathrm{~min}$ at $4{ }^{\circ} \mathrm{C}$, and the supernatants were analyzed using ultraviolet-visible light (UVvis) absorption spectrometers (JASCO V-550 or V-670, JASCO Corporation).

\section{Mechanical treatment of nanocelluloses}

Unless otherwise stated, a sonication-based mechanical treatment was performed on water dispersions of the artificial nanocelluloses $(30 \mathrm{ml}, 0.56$ $(\mathrm{w} / \mathrm{v}) \%)$ using a probe-type Sonifier 250 (Branson, Atsugi, Japan) for $2 \mathrm{~h}$ in an ice bath (power: $120 \mathrm{~W}$; duty cycle: $30 \%$ ). After the mechanical treatment, the dispersions were centrifuged at 15000 r.p.m. for $5 \mathrm{~min}$ at $4{ }^{\circ} \mathrm{C}$ to separately obtain the supernatants containing nanocelluloses that were stable in suspension even after centrifugation and precipitated nanocelluloses. These nanocelluloses were used in the hydrolysis experiments immediately after the mechanical treatment. The nanocellulose concentrations were estimated from the weights of the nanocelluloses after evaporation of water at $105^{\circ} \mathrm{C}$ for $48 \mathrm{~h}$.

\section{RESULTS AND DISCUSSION}

\section{Characterization of nanocelluloses synthesized by enzymatic} reactions

In a previous study, ${ }^{16}$ artificial sheet-like nanocelluloses with the cellulose II allomorph were synthesized by CDP-catalyzed enzymatic reactions using $\alpha \mathrm{G} 1 \mathrm{P}$ monomers and D-glucose primers. Although we have essentially followed their experimental methods, the CDP concentration was increased from $0.05 \mathrm{U} \mathrm{ml}^{-1}$ in the previous study to $0.2 \mathrm{U} \mathrm{ml}^{-1}$ in this study to increase the conversion of $\alpha \mathrm{G} 1 \mathrm{P}$ monomers to cellulose oligomers. The transparent reaction solutions became turbid dispersions after the enzymatic reactions, indicating the presence of water-insoluble products (Figure $2 \mathrm{a}$ ). The conversion was estimated to be $\sim 35 \%$ based on the mean DP of the products (see below). It is important to note that our experiments estimated the conversion to be $\sim 2 \%$ at $0.05 \mathrm{U} \mathrm{ml}^{-1}$. The TEM observations of the products revealed sheet-like morphologies with a width of several hundreds of nanometers and a length of less than several micrometers (Figure 2b), which were similar to those synthesized in a previous study. ${ }^{16}$

The NMR spectra of the products were adequately assigned to signals corresponding to cellulose oligomers according to results from previous studies (Figure 2c)..$^{18-20}$ The mean DP was estimated from the NMR spectra to be approximately 10 , which was similar to that obtained in a previous study. ${ }^{16}$ However, the matrix-assisted laser desorption/ionization time-of-flight mass spectra of the products indicated that the main DP was 8 (Supplementary Figure S1), which was slightly smaller than that estimated from the NMR spectra. A recent study ${ }^{21}$ similarly reported such differences. In this study, the previously mentioned conversion was estimated based on the mean DP from the NMR spectra, following to a previous paper. ${ }^{16}$ The attenuated total reflection-Fourier transform infrared absorption spectra contained two peaks at 3441 and $3489 \mathrm{~cm}^{-1}$ for the $\mathrm{OH}$ vibration bands corresponding to $2-\mathrm{OH} \cdots \mathrm{O}-6$ intramolecular hydrogen bonds, which were representative of the cellulose II allomorph (Figure 2d). ${ }^{22}$ The wide-angle X-ray diffraction measurements of the 

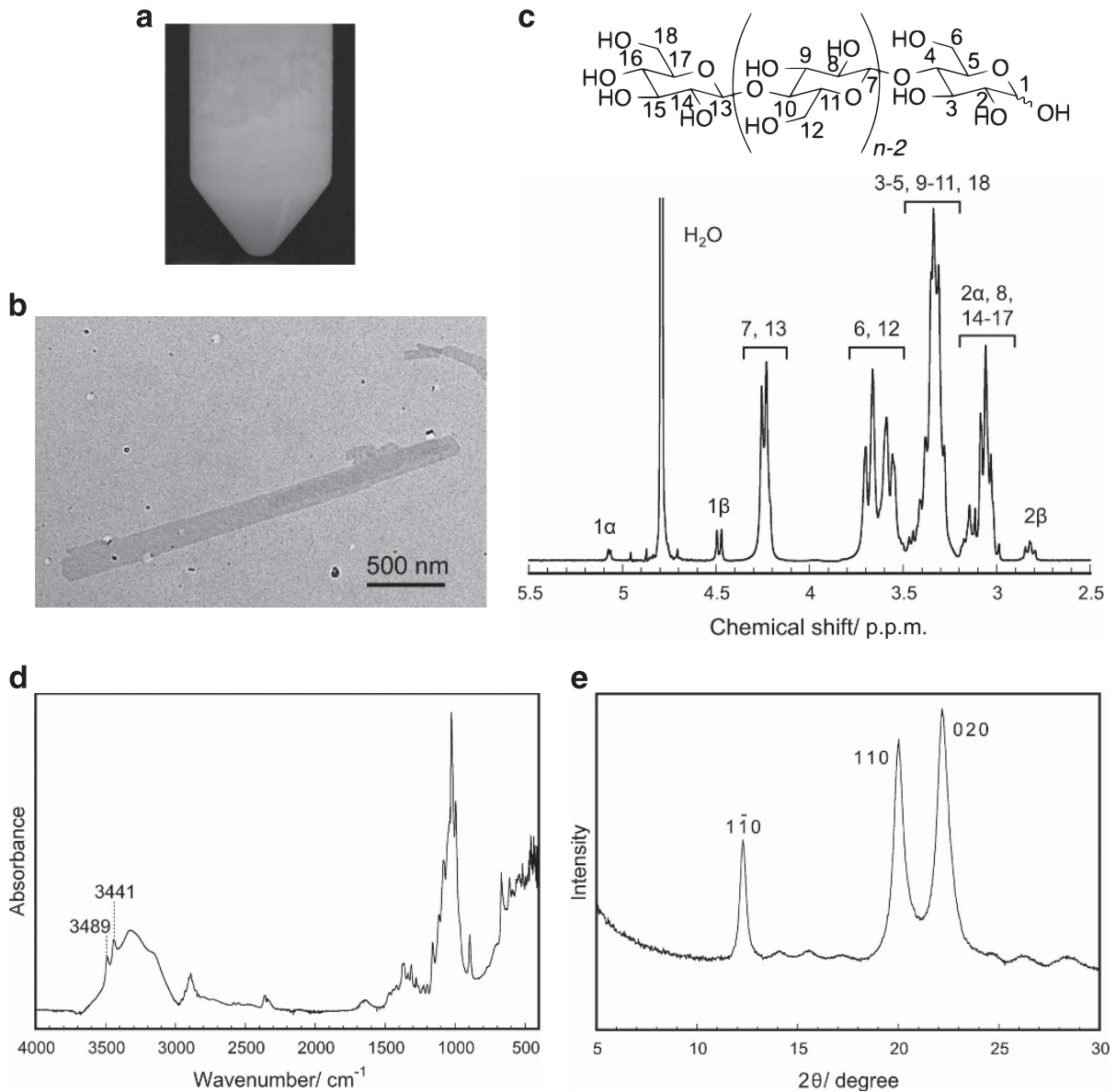

Figure 2 (a) Photo of turbid dispersions, (b) TEM image, (c) NMR spectrum, (d) ATR-FTIR absorption spectrum and (e) WAXD pattern of the as-prepared nanocelluloses. ATR-FTIR, attenuated total reflection-Fourier transform infrared; NMR, nuclear magnetic resonance; TEM, transmission electron microscopy; WAXD, wide-angle X-ray diffraction.

products confirmed the diffraction peaks assigned to $1 \overline{1} 0,110$ and 020 of the cellulose II allomorph (Figure $2 \mathrm{e}$ ). ${ }^{16}$ All of these observations indicated that the sheet-like nanocelluloses with the cellulose II allomorph were successfully synthesized via the CDP-catalyzed enzymatic reactions.

\section{Hydrolytic activities of the as-prepared nanocelluloses}

The hydrolytic activities of the artificial nanocelluloses were analyzed by hydrolysis of a model ester substrate (PNP) according to our previously reported method. ${ }^{10}$ The UV-vis absorption spectra of the reaction solutions after incubation for $7.5 \mathrm{~h}$ in the presence or absence of the nanocellulose contained absorption peaks at $\sim 400 \mathrm{~nm}$, which were assigned to the absorption of $p$-nitrophenolate ions and indicated the progress of the hydrolytic reactions (Figure 3a). The percent reactions were estimated to be $13 \%$ and $12 \%$ in the presence and absence of nanocelluloses, respectively (Figure $3 \mathrm{~b}$ ). The relative hydrolytic activity per nanocellulose weights was estimated to be $0.6 \%$ per mg using the increment of the percent reactions $(\sim 1 \%)$ and the nanocellulose concentration. Therefore, the as-prepared nanocelluloses slightly accelerated the hydrolysis of PNP. However, the hydrolytic activities were much smaller than that of nature-based NCC derived from tunicate, which exhibited a percent reaction of $47 \%$ under the same conditions. ${ }^{10}$ Therefore, we attempted to improve the hydrolytic activities of the artificial nanocelluloses in the next step.

\section{Characterization of mechanically treated nanocelluloses}

In our previous studies, ${ }^{10,11}$ we proposed that the hydrolytic activities of nature-based NCC may be derived from the activated OH groups of the repeating D-glucose units on the outermost surface of NCC. However, because cellulose oligomers are aligned perpendicularly to the surface of the sheet-like nanocelluloses synthesized in this study, relatively few $\mathrm{OH}$ groups of the repeating D-glucose units are exposed on the outermost surface. Therefore, to improve the hydrolytic activities, the sheet-like nanocelluloses were mechanically crushed into smaller pieces with relatively larger surface areas using a sonication treatment. The nanocellulose at $0.56(\mathrm{w} / \mathrm{v}) \%$ after the sonication treatment was separated into supernatant and precipitate through centrifugation. The supernatant concentration was estimated to be $0.011(\mathrm{w} / \mathrm{v}) \%$. Therefore, the precipitate concentration was assumed to be $\sim 0.55(\mathrm{w} / \mathrm{v}) \%$. The TEM images of the nanocelluloses in the supernatants and precipitates indicated that distorted and smaller nanocelluloses with sizes of less than several hundreds of nanometers were obtained from the sonication treatment (Figure 4a). In fact, the nanocelluloses in the supernatants appeared to be more dispersed than those in the precipitates.

The NMR spectra of the nanocelluloses in the supernatants and precipitates contained representative signals for cellulose oligomers (Figure 4b) and revealed a mean DP of 7 and 10, respectively. The matrix-assisted laser desorption/ionization time-of-flight mass spectra of the nanocelluloses in the supernatants indicated that the main DP 
was 6, which confirmed the results obtained from the NMR spectra (Figure 4c). However, matrix-assisted laser desorption/ionization time-of-flight mass spectra of the nanocelluloses in the precipitates revealed that the main DP slightly increased compared with that of the original nanocelluloses. In addition, the spectra were broadened (Figure 4c). These observations suggested that the sonication treatment of the as-prepared nanocelluloses was unexpectedly good at extracting the water-dispersible nanocelluloses with smaller molecular
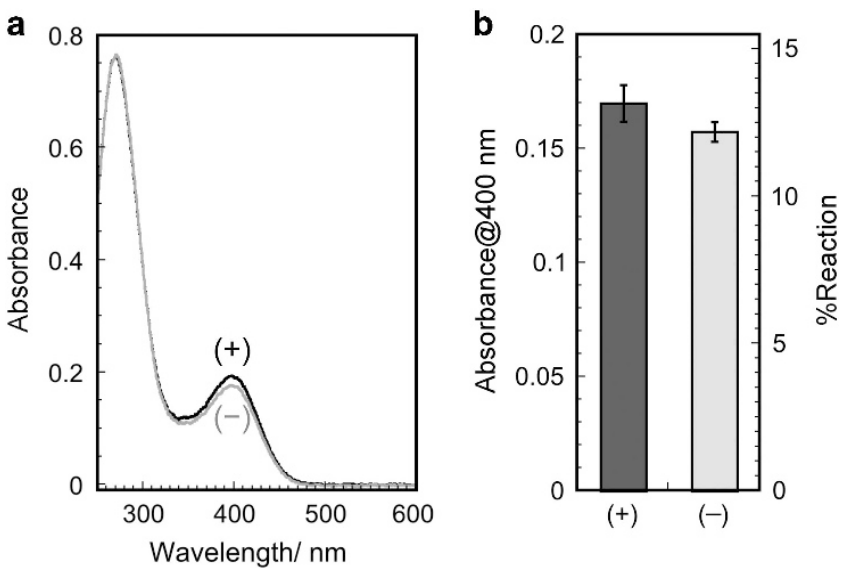

Figure 3 (a) UV-vis absorption spectra of the reaction solutions after incubation for $7.5 \mathrm{~h}$ in the presence $(+)$ and absence $(-)$ of the as-prepared nanocelluloses and (b) the percent reactions. UV-vis, ultraviolet-visible light. weights. The attenuated total reflection-Fourier transform infrared absorption spectra of the nanocelluloses in the supernatants and precipitates contained two peaks corresponding to $\mathrm{OH}$ vibration bands, which were assigned to the cellulose II allomorph (Figure $4 \mathrm{~d}){ }^{22}$ However, the peaks of the nanocelluloses in the supernatants were relatively weak and broad, suggesting lower crystallinity compared with that of the precipitated and as-prepared nanocelluloses. Although it is difficult to reasonably explain the mechanism for such selective extraction, the nanocelluloses in the supernatants exhibited unique hydrolytic activities.

\section{Hydrolytic activities of mechanically treated nanocelluloses}

The hydrolytic activities of the nanocelluloses in the supernatants and precipitates were analyzed by PNP hydrolysis. The UV-vis absorption spectra of the reaction solutions after incubation for $7.5 \mathrm{~h}$ in the presence of the nanocelluloses contained absorption peaks for the $p$ nitrophenolate ions at $\sim 400 \mathrm{~nm}$, indicating the progress of the hydrolytic reactions (Figure 5a). Significantly, the percent reaction for hydrolysis by the nanocelluloses in the supernatants was estimated to be $21 \%$ (Figure $5 \mathrm{~b}$ ), which was greater than that for the as-prepared nanocelluloses, even though the former concentration was 50 times smaller than the latter. In fact, the relative hydrolytic activity per nanocellulose weight was estimated to be $0.3 \%$ per $\mu$ g using the increment of percent reactions ( $\sim 9 \%)$ and the nanocellulose concentration, and this activity was 500 times greater than the previously mentioned activity of the as-prepared nanocelluloses ( $0.6 \%$ per $\mathrm{mg}$ ). Therefore, the nanocelluloses in the supernatants exhibited much greater hydrolytic activity than those of the as-prepared nanocelluloses. a
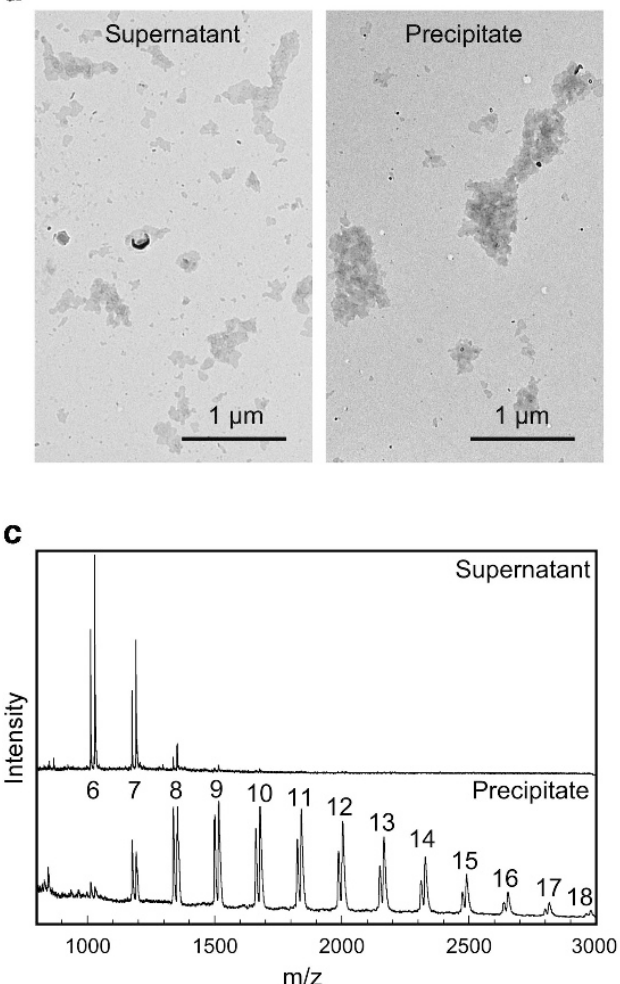

b

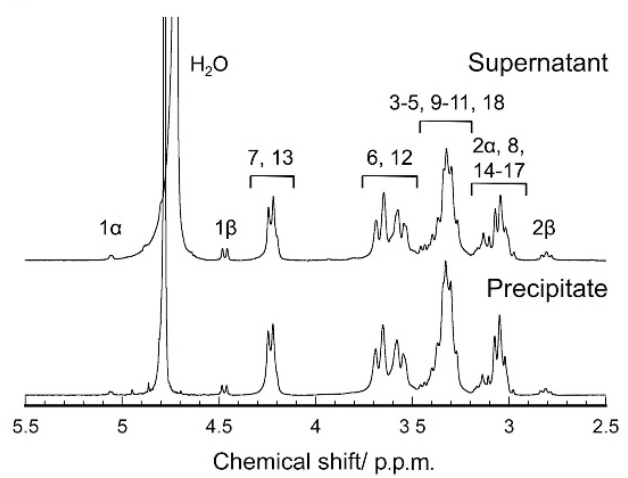

d

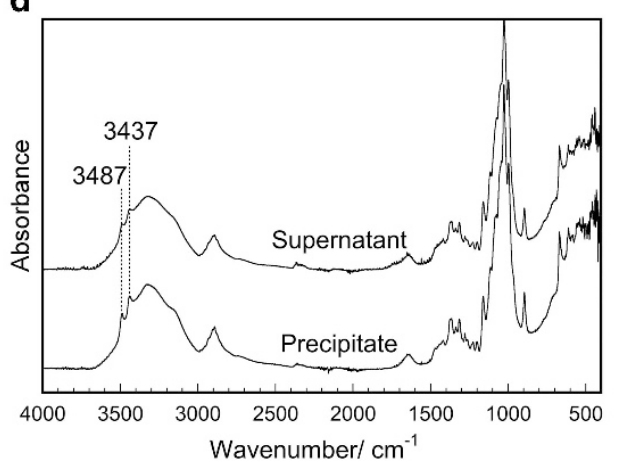

Figure 4 (a) TEM images, (b) NMR spectra (see the chemical structure of cellulose in Figure 2c), (c) MALDI-TOF-MS spectra and (d) ATR-FTIR absorption spectra of the mechanically treated nanocelluloses in the supernatants and precipitates after centrifugation. ATR-FTIR, attenuated total reflection-Fourier transform infrared; MALDI-TOF-MS, matrix-assisted laser desorption/ionization time-of-flight mass; NMR, nuclear magnetic resonance; TEM, transmission electron microscopy. 
The time dependence of the PNP hydrolysis by the nanocelluloses was determined to follow a pseudo-first order reaction with correlation coefficients of more than 0.998 (Figure $5 \mathrm{c}$ ). The rate constant was estimated to be $0.029 \mathrm{~h}^{-1}$, which was 2.6 times greater than that for spontaneous reactions $\left(0.011 \mathrm{~h}^{-1}\right)$. However, the nanocelluloses in the
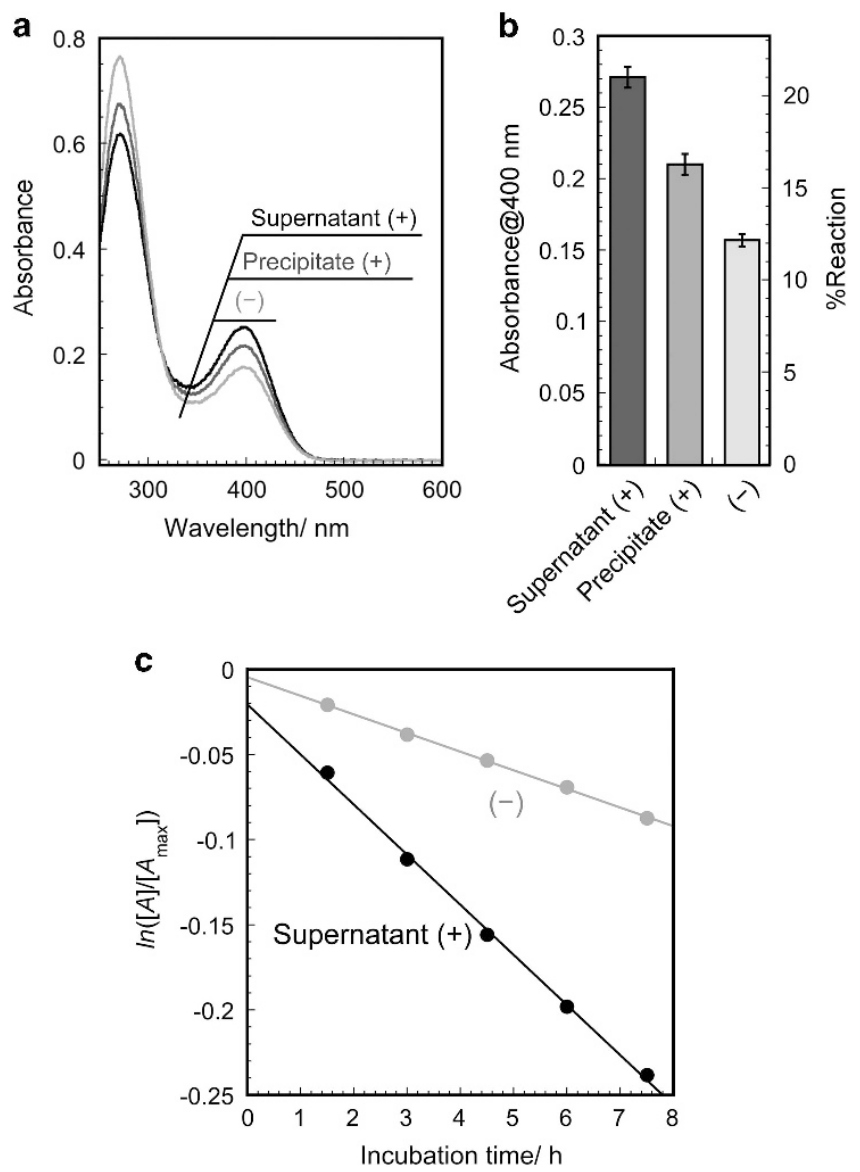

Figure 5 (a) UV-vis absorption spectra of the reaction solutions after incubation for $7.5 \mathrm{~h}$ in the presence $(+)$ and absence $(-)$ of mechanically treated nanocelluloses, (b) percent reactions and (c) fitting to a pseudo-first order reaction.

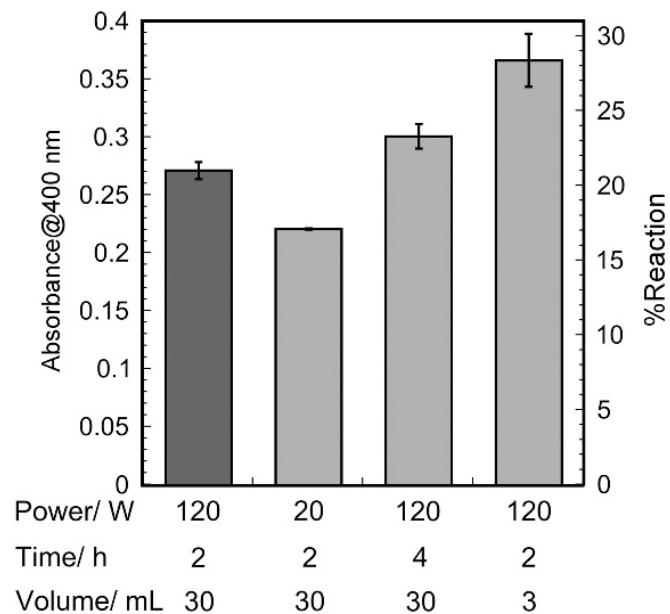

Figure 6 Effects of sonication conditions on the hydrolytic activities of mechanically treated nanocelluloses in the supernatants. precipitates exhibited intermediate activities (Figure 5b). These observations suggest that the mechanically treated nanocelluloses with relatively larger surface areas exhibit greater hydrolytic activities than those of the as-prepared nanocelluloses.

An increase in the sonication power from 20 to $120 \mathrm{~W}$ or sonication time from 2 to $4 \mathrm{~h}$ along with a decrease in the volume of the dispersions from 30 to $3 \mathrm{ml}$ increased the hydrolytic activities of the nanocelluloses in the supernatants (Figure 6). These observations confirmed that the mechanical treatment was essential for obtaining active nanocelluloses. However, the commercially available cellohexaose did not exhibit hydrolytic activities even though the nanocelluloses in the supernatant had a main DP of 6 (Supplementary Figure S2). This observation suggests that the nanocelluloses in the supernatants have unique nanostructures that are appropriate for PNP hydrolysis. It is important to note that the hydrolytic activities of the nanocelluloses in the supernatants did not change even after autoclave treatment at $121{ }^{\circ} \mathrm{C}$ for $20 \mathrm{~min}$ (Supplementary Figure S2), suggesting that unfavorable contamination of the enzymes did not occur. It is also important to note that the hydrolytic activities of the nanocelluloses in the supernatants did not change in the presence of a chelating reagent (EDTA) at $2 \mathrm{~mm}$ (Supplementary Figure S2), suggesting that unfavorable contamination of metal ions did not occur. On the other hand, the active nanocelluloses were repetitively extracted from the same nanocelluloses by sonication treatment (Supplementary Figure S3). In fact, the nanocelluloses with similar activities were extracted until four sonication treatments were performed, and then, the activities decreased gradually.

\section{Hydrolytic activities of concentrated nanocelluloses}

To obtain more active dispersions of nanocelluloses, the supernatants obtained after sonication treatment were concentrated 10 times through water evaporation, and then, the obtained dispersions were diluted into appropriate concentrating ratios. The hydrolytic activities were similarly analyzed by PNP hydrolysis (Figure 7). The percent reactions after $7.5 \mathrm{~h}$ of reaction increased with as the concentrating ratio increased and reached $51 \%$ for 10 times more concentrated nanocelluloses at $0.11(\mathrm{w} / \mathrm{v}) \%$, and the resulting percent reaction was comparable to that of nature-based NCC at $0.5(\mathrm{w} / \mathrm{v}) \% .{ }^{10}$ Therefore, an increase in the concentration of nanocellulose based on water evaporation produced more active dispersions. However, when we compared the increase in yields by subtracting the yield of

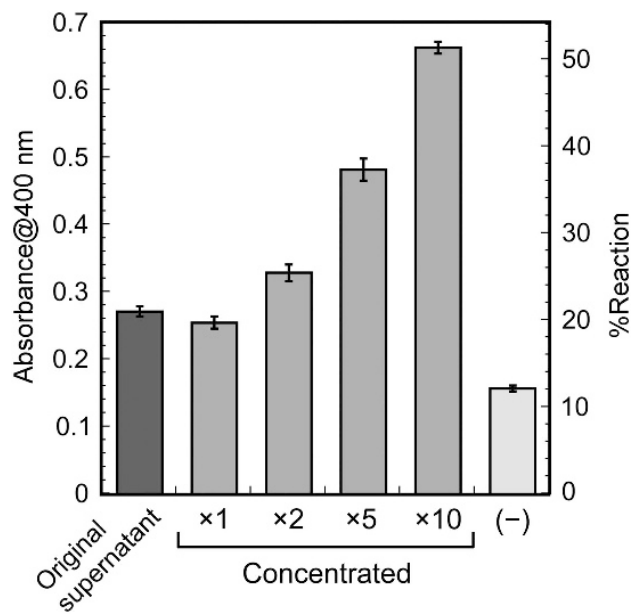

Figure 7 PNP hydrolysis by nanocelluloses in concentrated supernatants after $7.5 \mathrm{~h}$ of reaction. PNP, p-nitrophenyl acetate. 
spontaneous hydrolysis, the increment for the 10 times more concentrated nanocelluloses was estimated to be 5.3. Ideally, this value should be 10 . Similarly, the activities of other concentrated nanocelluloses were smaller than those expected from the concentrating ratios. This discrepancy is most likely derived from unfavorable aggregation of the nanocelluloses during the concentrating process.

The 10 times more concentrated nanocelluloses were used for hydrolysis of other substrates, such as disodium $p$-nitrophenyl phosphate and $p$-nitroacetanilide, and the hydrolyses were similarly analyzed by absorption of $p$-nitrophenolate and $p$-nitroaniline products at 400 and $410 \mathrm{~nm}$, respectively. The reactions were performed for 1 week because the hydrolyses of both substrates were observed with nature-based NCC. ${ }^{10}$ Unfortunately, hydrolysis was barely observed with the concentrated nanocelluloses (Supplementary Figure S4). When an ester substrate (PNP) was further added into the reaction solutions after the 1 week reaction, hydrolysis did not proceed (data not shown). Therefore, the concentrated nanocelluloses may have lost their hydrolytic activities due to unfavorable aggregation of the nanocelluloses during the 1 week reaction. Therefore, the present nanocelluloses exhibited limited potential for hydrolysis of the activated ester linkages of small organic molecules, such as PNP.

\section{CONCLUSIONS}

Artificial sheet-like nanocelluloses with the cellulose II allomorph were synthesized via CDP-catalyzed enzymatic reactions using $\alpha \mathrm{G} 1 \mathrm{P}$ monomers and D-glucose primers. The mean DP of the component cellulose oligomers was estimated to be $\sim 10$ based on NMR measurements. The as-prepared nanocelluloses exhibited relatively low but credible hydrolytic activities against a model ester substrate (PNP). Sonication-based mechanical treatment of the as-prepared nanocelluloses produced distorted and smaller nanocelluloses composed of cellulose oligomers with a relatively smaller DP. The mechanically treated nanocelluloses in the supernatants exhibited significantly higher hydrolytic activities even though the concentrations of nanocelluloses were much smaller than those of the asprepared nanocelluloses. More active dispersions of the nanocelluloses were produced by evaporation of the water solvent. This study revealed that enzymatically synthesized artificial nanocelluloses with the cellulose II allomorph had the potential to exhibit hydrolytic activities against small organic substrates, which is similar to naturebased NCC with the cellulose I allomorph. Our results will contribute to the understanding and development of saccharide-based artificial hydrolases in the near future.

\section{CONFLICT OF INTEREST}

The authors declare no conflict of interest.

\section{ACKNOWLEDGEMENTS}

We thank the Center for Advanced Materials Analysis (Tokyo Tech) for the TEM observations and wide-angle X-ray diffraction measurements. This study was partially supported by the Funding Program for Next Generation World-
Leading Researchers (NEXT Program) and Grants-in-Aids for Scientific Research (26288056 and 26620174) from the Japan Society for the Promotion of Science.

1 Habibi, Y., Lucia, L. A. \& Rojas, O. J. Cellulose nanocrystals: chemistry, self-assembly, and applications. Chem. Rev. 110, 3479-3500 (2010).

2 Domingues, R. M. A., Gomes, M. E. \& Reis, R. L. The potential of cellulose nanocrystals in tissue engineering strategies. Biomacromolecules 15, 2327-2346 (2014).

3 Lin, N., Huang, J. \& Dufresne, A. Preparation, properties and applications of polysaccharide nanocrystals in advanced functional nanomaterials: a review. Nanoscale 4, 3274-3294 (2012).

4 Moon, R. J., Martini, A., Nairn, J., Simonsen, J. \& Youngblood, J. Cellulose nanomaterials review: structure, properties and nanocomposites. Chem. Soc. Rev. 40, 3941-3994 (2011).

5 Samir, M., Alloin, F. \& Dufresne, A. Review of recent research into cellulosic whiskers, their properties and their application in nanocomposite field. Biomacromolecules 6 , 612-626 (2005)

6 Klemm, D., Kramer, F., Moritz, S., Lindström, T., Ankerfors, M., Gray, D. \& Dorris, A. Nanocelluloses: a new family of nature based materials. Anegw. Chem. Int. Ed. Engl. 50, 5438-5466 (2011).

7 Henriksson, M., Berglund, L. A., Isaksson, P., Lindström, T. \& Nishino, T. Cellulose nanopaper structures of high toughness. Biomacromolecules $\mathbf{9}$, 1579-1585 (2008)

8 Dong, X. M., Kimura, T., Revol, J. F. \& Gray, D. G. Effects of ionic strength on the isotropic-chiral nematic phase transition of suspensions of cellulose crystallites. Langmuir 12, 2076-2082 (1996).

9 Samir, M. A. S. A., Chazeau, L., Alloin, F., Cavaille, J.-Y., Dufresne, A. \& Sanchez, J.-Y. Poe-based nanocomposite polymer electrolytes reinforced with cellulose whiskers. Electrochim. Acta 50, 3897-3903 (2005).

10 Serizawa, T., Sawada, T., Okura, H. \& Wada, M. Hydrolytic activities of crystalline cellulose nanofibers. Biomacromolecules 14, 613-617 (2013).

11 Serizawa, T., Sawada, T. \& Wada, M. Chirality-specific hydrolysis of amino acid substrates by cellulose nanofibers. Chem. Commun. 49, 8827-8829 (2013).

12 Kobayashi, S., Shoda, S.-I. \& Uyama, H. Enzymatic polymerization and oligomerization. Adv. Polym. Sci. 121, 1-30 (1995).

13 Kobayashi, S., Sakamoto, J. \& Kimura, S. In vitro synthesis of cellulose and related polysaccharides. Prog. Polym. Sci. 26, 1525-1560 (2001).

14 Kadokawa, J.-I. Precision polysaccharide synthesis catalyzed by enzymes. Chem. Rev. 111, 4308-4345 (2011).

15 Samain, E., Lancelon-Pin, C., Férigo, F., Moreau, V., Chanzy, H., Heyraud, A. \& Driguez, H. Phosphorolytic synthesis of cellodextrins. Carbohydr. Res. 217, 217-226 (1995).

16 Hiraishi, M., Igarashi, K., Kimura, S., Wada, M., Kitaoka, M. \& Samejima, M. Synthesis of highly ordered cellulose II in vitro using cellodextrin phosphorylase. Carbohydr. Res. 344, 2468-2473 (2009).

17 Yataka, Y., Sawada, T. \& Serizawa, T. Enzymatic synthesis and post-functionalization of two-dimensional crystalline cellulose oligomers with surface-reactive groups. Chem. Commun. 51, 12525-12528 (2015).

18 Flugge, L. A., Blank, J. T. \& Petillo, P. A. Isolation, modification, and NMR assignments of a series of cellulose oligomers. J. Am. Chem. Soc. 121, 7228-7238 (1999)

19 Xiong, B., Zhao, P., Cai, P., Zhang, L., Hu, K. \& Cheng, G. NMR spectroscopic studies on the mechanism of cellulose dissolution in alkali solutions. Cellulose 20, 613-621 (2013).

20 Sugiyama, H., Hisamichi, K., Usui, T., Sakai, K. \& Ishiyama, J. I. A study of the conformation of $\beta$-1,4-linked glucose oligomers, cellobiose to cellohexaose, in solution. J. Mol. Struct. 556, 173-177 (2000).

21 Petrović, D. M., Kok, I., Woortman, A. J. J., Ćirić, J. \& Loos, K. Characterization of oligocellulose synthesized by reverse phosphorolysis using different cellodextrin phosphorylases. Anal. Chem. 87, 9639-9646 (2015).

22 Horikawa, Y., Konakahara, N., Imai, T., Abe, K., Kobayasi, Y. \& Sugiyama, J. The structural changes in crystalline cellulose and effects on enzymatic digestibility. Polym. Degrad. Stab. 98, 2351-2356 (2013).

Supplementary Information accompanies the paper on Polymer Journal website (http://www.nature.com/pj) 